\section{Hit and flip}

New J.Phys. (in the press)

Next time you grab a badminton racket for a casual game you might want to try some new tricks physicists have come up with. Caroline Cohen and colleagues have developed an aerodynamic model to understand the unique flight pattern of the shuttlecock and what this means for the game.

The winning advantage in badminton is in accurate control of the shuttlecock trajectory and as Cohen et al. point out, it's all about geometry. The shuttlecock can reach speeds of over $130 \mathrm{~m} \mathrm{~s}^{-1}$ and it will always hit the racket cork-first, meaning that it will always flip after impact. This is the result of an aerodynamic torque caused by the interplay between gravity and drag, which in turn occurs because of the shifted centres of mass and pressure.

Cohen and colleagues found that an opening angle of around 45 degrees minimizes the flipping time. The shuttlecock rotates around its axis because of the asymmetry of its skirt and the choice of material turns out to be quite important. Feather skirts have a slightly larger drag than plastic ones, but also higher rotation speeds - both very important in controlling the trajectory.

\section{Balancing act}

Phys. Rev. X 5, 021012 (2015)

Charged colloidal particles repel or attract, depending on the signs of their charges. But the underlying forces are not necessarily governed by the standard Coulomb law. In a solvent with ions, the charge of a colloid gets shielded and is felt only weakly beyond a certain distance, known as the screening length. So, by cleverly choosing the solvent, the screened Coulomb, or Yukawa, potential of a colloid can be tuned.

Ahmet Demirörs and colleagues have now exploited charge screening to create particular colloidal clusters. The authors used binary mixtures of small and large oppositely charged, micrometre-sized particles, and observed clusters where a small particle is surrounded by up to six large satellite particles. These 'colloidal molecules' are the analogues of, for example, methane or phosphorus pentachloride.

Demirörs et al. identified the criteria for such clusters to self-assemble and remain stable: in addition to an excess of satellite particles, the right balance has to be reached between attraction and repulsion throughout the 'molecule'. A promising feature of the fabrication scheme is that a given number of satellite particles can be obtained for different core sizes, providing a handle on surface coverage and patchiness.

$B V$

\section{When we were young \\ Astrophys. J 804, L30 (2015)}

Things were different when the Universe was young. In fact, stars used to form much faster 13 billion years ago, according to a study by Pascal Oesch and co-workers. Within a mere 670 million years, EGS-zs8-1 in the constellation Boötes had amassed more than $15 \%$ of the total mass of the Milky Way.

The team used a combination of the Keck Observatory on Mauna Kea in Hawaii and the Spitzer and Hubble space telescopes to probe the most distant parts of the Universe. Their spectroscopic measurements of EGS-zs8-1 confirm that it is the brightest and most distant galaxy (apart from a gamma-ray burst $630 \mathrm{Myr}$ after the Big Bang). Crucially, this source dates back to the 'cosmic reionization epoch', when interstellar neutral hydrogen became ionized, and which

\section{Know the ropes}

Nature Commun. 6, 7008 (2015)

Although plasmas are the most abundant state of ordinary matter in the Universe, they are not known for their stability. This can be seen in our nearby star, which regularly ejects bursts of gases and magnetic fields that can cause severe damage to satellites, power grids and radio communications. Techniques to predict such bursts could help minimize the effects of future events, but greater understanding of their formation are required to develop such methods. The new solar telescope at the Big Bear Solar Observatory has now provided images of unprecedented resolution featuring magnetic flux ropes - one of the elements thought to play a crucial role in a range of solar eruptions.

Flux ropes are helical, current-carrying systems of twisted magnetic fields that rotate around a common axis. They are well known from laboratory plasma physics experiments, but identifying their presence in the Sun has proved challenging. The $1.6 \mathrm{~m}$ aperture of the new solar telescope has provided detailed images of the structure and evolution of a flaring twisted flux rope in the low solar atmosphere, bringing the relationship between flux ropes and solar eruptions within reach. has been challenging to study. The massive young stars formed rapidly and interacted with the primordial gas.

The James Webb Space Telescope, due to launch in 2018, will help us delve further into our past to clarify the composition and formation of the early Universe.

\section{Cell swell}

Phys. Rev. Lett. 114, 208101 (2015)

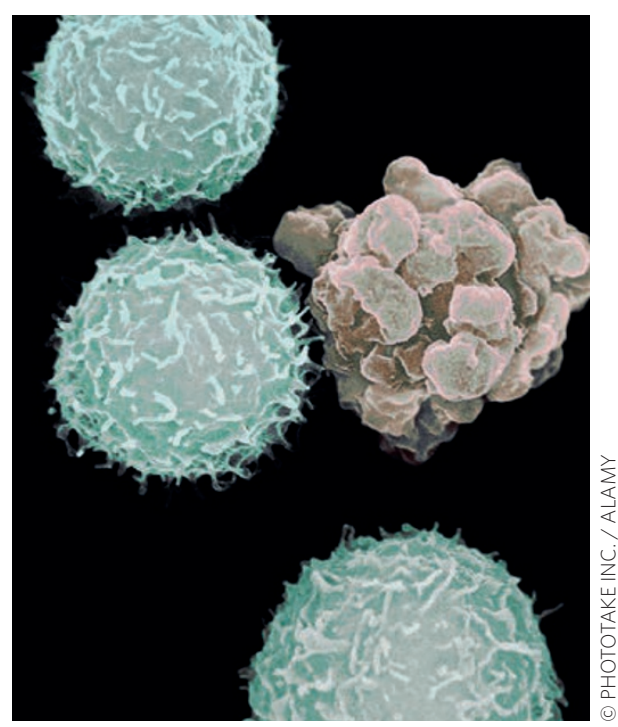

Cells programmed to die undergo what's known as blebbing: the thin layer of crosslinked biopolymers underlying the membrane ruptures, causing it to bulge outwards in transient fluid-filled protrusions (pictured). These shape changes also occur during cell division and migration, and are generally thought to be driven by pressure-induced flow inside the cell without radically modifying its volume. But now Alessandro Taloni and colleagues have observed fluid transport through the membrane of migrating stem cells, and found evidence to suggest that it facilitates blebbing.

The authors developed a way of imaging that allowed them to observe water flow in and out of the cells. They noted a marked reduction in bleb activity when this flow was hindered, and a correlation between surface and volume fluctuations. Together with simulations of fluid-mediated deformations, this led them to infer a link between blebbing and the fluctuations induced by water transport. Both experimental and numerical results seem to be incompatible with the oft-held assumption of conserved volume via an impermeable membrane - which may breathe new life into the study of cell death. 